\title{
Finite Element Solution of the Fundamental Equations of Semiconductor Devices. I
}

\author{
By Miloš Zlámal
}

\author{
Dedicated to Professor Joachim Nitsche on the occasion \\ of the sixtieth anniversary of his birthday
}

\begin{abstract}
We investigate the nonstationary equations of the semiconductor device theory consisting of a Poisson equation for the electric potential $\psi$ and of two highly nonlinear continuity equations for carrier densities $n$ and $p$. We use simplicial elements with linear polynomials and four-node two-dimensional and eight-node three-dimensional isoparametric elements. There are constructed finite element solutions such that the current densities $\mathbf{J}_{n}, \mathbf{J}_{p}$ and the electric field strength $\|\nabla \psi\|$ are constant on each element. Two schemes are proposed: one is nonlinear, the other is partly linear. The schemes preserve the property of the exact solution (corresponding to the physical meaning) that the carrier densities $n$ and $p$ are positive. Existence of the solution is proved in both cases, unicity in the second case. A subsequent paper II will be devoted to problems of stability and convergence.
\end{abstract}

1. Introduction. We consider a system of three equations in a bounded domain $\Omega \subset R^{m}, m=2,3$, which form the basic model of mobile carrier transport in a semiconductor device (see, e.g., Buturla, Cottrell, Grossman, Salsburg [1]):

$$
-\Delta \psi=\frac{q}{\varepsilon}(p-n+N(x))
$$

$$
\begin{aligned}
& \frac{\partial n}{\partial t}=\nabla \cdot\left[D_{n}(x,\|\nabla \psi\|) \nabla n-\mu_{n}(x,\|\nabla \psi\|) n \nabla \psi\right]-R_{n}(n, p), \\
& \frac{\partial p}{\partial t}=\nabla \cdot\left[D_{p}(x,\|\nabla \psi\|) \nabla p+\mu_{p}(x,\|\nabla \psi\|) p \nabla \psi\right]-R_{p}(n, p) .
\end{aligned}
$$

The unknowns are the electrostatic potential $\psi$ and the electron and hole densities $n$ and $p$. All coefficients appearing in (1.1)-(1.3) are positive. $q$ and $\varepsilon$ are constants ( $q$ is the electron charge, $\varepsilon$ is the dielectric permittivity), the diffusion coefficients $D_{s}(x, \xi), s=n, p$, are related to the mobilities $\mu_{s}(x, \xi)$ by the Einstein relation $D_{s}(x, \xi)=U_{T} \mu_{s}(x, \xi)$ where $U_{T}$ is the thermal voltage. $N(x)$ is a given function of $x=\left(x_{1}, \ldots, x_{m}\right)$ which is equal to $N_{D}(x)-N_{A}(x), N_{D}(x)$ and $N_{A}(x)$ being the donor and acceptor impurity densities. $N$ changes its values extremely rapidly if $x$ approaches the so-called junctions (curves or surfaces lying in $\Omega$ ). Close to junctions, $\max \|\nabla N\|$ assumes values $10^{18}-10^{23} \mathrm{~cm}^{-4} \cdot R_{s}(n, p)$ are recombination terms. We

Received November 16, 1983; revised December 19, 1983, January 23, 1984, October 3, 1984 and March 29, 1985.

1980 Mathematics Subject Classification. Primary 65N30. 
simplify the system, taking

$$
R_{n}(n, p)=R_{p}(n, p)=R(n, p)=\frac{n p-n_{i}^{2}}{\tau_{p}\left(n+n_{i}\right)+\tau_{n}\left(p+n_{i}\right)}
$$

and further $\tau_{n}=\tau_{p}=\tau$. These simplifications are not essential for the construction of the approximate solution as well as for all results of this paper. Let us remark at this place that in practical computations $\tau$ is of the magnitude $10^{-6} \mathrm{~s}$ and the length $t_{0}$ of the time interval is $t_{0}=k \tau$, where $1 \leqslant k \leqslant 10$.

Before formulating the problem completely, we scale the system by introducing new dependent as well as independent dimensionless variables and the new dimensionless doping profile and mobilities,

$$
\begin{gathered}
\psi=U_{T} \bar{\psi}, \quad n=n_{i} \bar{n}, \quad p=n_{i} \bar{p}, \quad x_{j}=l \bar{x}_{j} \quad(j=1, \ldots, m), t=\tau \bar{t}, \\
N=n_{i} \bar{N}, \quad \mu_{s}=\mu_{0} \bar{\mu}_{s}, \quad s=n, p .
\end{gathered}
$$

Here $l=5 \times 10^{-4} \mathrm{~cm}$ is the realistic value of $\operatorname{diam}(\Omega), n_{i}=10^{10} \mathrm{~cm}^{-3}$ is the intrinsic number, and for silicon at room temperature $\mu_{0}=1300 \mathrm{~cm}^{2} / \mathrm{Vs}$ for electrons and $\mu_{0}=500 \mathrm{~cm}^{2} / \mathrm{Vs}$ for holes. Keeping the same notation of all variables, of the mobilities and of the doping profile as before, we get

$$
\begin{gathered}
-\Delta \psi=\alpha(p-n+N(x)) \quad \text { in } R \forall t \in(0, T), \\
\left.\frac{\partial n}{\partial t}=\gamma_{n} \nabla \cdot\left\{\mu_{n}(x,\|\nabla \psi\|)[\nabla n-n \nabla \psi]\right\}-R(n, p)\right\} \\
\left.\frac{\partial p}{\partial t}=\gamma_{p} \nabla \cdot\left\{\mu_{p}(x,\|\nabla \psi\|)[\nabla p+p \nabla \psi]\right\}-R(n, p)\right\} \\
R(n, p)=\frac{n p-1}{n+p+2}, \quad Q=\Omega \times(0, T) .
\end{gathered}
$$

The values of $\alpha=l^{2} q n_{i} / \varepsilon U_{T}, \quad \gamma_{s}=\tau U_{T} \mu_{0} / l^{2}(s=n, p)$ and $T$ are $\alpha=0.016$, $\gamma_{n}=130, \gamma_{p}=50, T \in[1,10]$. The quantity sup $\alpha\|\nabla N\|$ is of the order $10^{3}-10^{8}$.

Let us remark that the expressions in the brackets on the right-hand sides of (1.2) and (1.3) are equal to $\mathbf{J}_{n}$ and $-\mathbf{J}_{p}$, respectively, where

$$
\mathbf{J}_{n}=q \mu_{n}(x,\|\nabla \psi\|)\left(U_{T} \nabla n-n \nabla \psi\right), \quad \mathbf{J}_{p}=-q \mu_{p}(x,\|\nabla \psi\|)\left(U_{T} \nabla p+p \nabla \psi\right) .
$$

$\mathbf{J}_{n}$ and $\mathbf{J}_{p}$ are the electron and hole current densities. In the scaled system the current densities are

$$
\begin{aligned}
& \mathbf{J}_{s}=\beta_{s} \mathbf{J}_{s}^{*} \quad(s=n, p), \\
& \beta_{s}=\frac{q U_{T} n_{i}}{l} \mu_{0}\left(\beta_{n}=1.04 \times 10^{-4} A \mathrm{~cm}^{-2}, \beta_{p}=4 \times 10^{-5} A \mathrm{~cm}^{-2}\right),
\end{aligned}
$$

where $\mathbf{J}_{n}^{*}$ and $\mathbf{J}_{p}^{*}$ are dimensionless quantities,

$$
\mathbf{J}_{n}^{*}=\mu_{n}(x,\|\nabla \psi\|)(\nabla n-n \nabla \psi), \quad \mathbf{J}_{p}^{*}=-\mu_{p}(x,\|\nabla \psi\|)(\nabla p+p \nabla \psi) .
$$

The first terms on the right-hand sides of (1.6) and (1.7) are equal to $\gamma_{n} \nabla \cdot \mathbf{J}_{n}^{*}$ and $-\gamma_{p} \nabla \cdot \mathbf{J}_{p}^{*}$, respectively. 
We denote by $\Gamma=\Gamma^{1} \cup \Gamma^{2}$ the boundary of $\Omega$. The basic boundary conditions are Dirichlet nonhomogeneous and Neumann homogeneous boundary conditions

$$
\begin{aligned}
& \left.\psi\right|_{\Gamma^{1}}=\left.\psi^{*}\right|_{\Gamma^{1}},\left.\quad n\right|_{\Gamma^{1}}=\left.n^{*}\right|_{\Gamma^{1}},\left.\quad p\right|_{\Gamma^{1}}=\left.p^{*}\right|_{\Gamma^{1}}, \\
& n^{*}(x), p^{*}(x)>0 \text { on } \Gamma^{1} \\
& \left.\frac{\partial \psi}{\partial \nu}\right|_{\Gamma^{2}}=\left.\frac{\partial n}{\partial \nu}\right|_{\Gamma^{2}}=\left.\frac{\partial p}{\partial \nu}\right|_{\Gamma^{2}}=0 \\
& \text { and } \left.\quad \psi\left(x^{0}, t\right)=0, \quad x^{0} \in \bar{\Omega} \text { if } \Gamma^{1}=\varnothing\right)
\end{aligned}
$$

Here $\psi^{*}(x), n^{*}(x), p^{*}(x)$ are sufficiently smooth functions defined on $\bar{\Omega}$ (of course, for computations we need to know the values of these functions on $\Gamma^{1}$ only).

In addition, we have an initial condition

(1.12) $n=n^{0}(x), \quad p=p^{0}(x)$ in $\Omega, \quad n^{0}(x), p^{0}(x)>0 \quad$ on $\bar{\Omega}$.

In case that $\Gamma^{1}=\varnothing$ we need the compatibility condition

$$
\int_{\Omega}\left[p^{0}-n^{0}+N\right] d x=0 \text { if } \Gamma^{1}=\varnothing .
$$

The last requirement is

$$
n(x, t)>0, \quad p(x, t)>0 \quad \text { on } \bar{Q} .
$$

Remark 1.1. We can easily prove that any sufficiently smooth functions $\psi, n, p$ satisfying (1.5)-(1.7) and (1.10)-(1.12) satisfy automatically (1.14) if $\mu_{s}(x, \xi)$ are continuously differentiable functions.

We formulate the problem (1.5)-(1.7), (1.10)-(1.14) in a variational form. We denote by $V$ the space

$$
V=\left\{v \in H^{1}(\Omega),\left.v\right|_{\Gamma^{1}}=0\right\} .
$$

Let us remark that we use the usual notations of the Sobolev spaces $H^{m}(\Omega), m=1$, $2, \ldots$, and $H^{m, p}(\Omega), m=1,2, \ldots, 1 \leqslant p \leqslant \infty\left(H^{m, 2}=H^{m}\right)$. Also $L^{p}(\Omega), 1 \leqslant p$ $\leqslant \infty$ are the Lebesgue spaces and $(\cdot, \cdot)$ will denote the scalar product in $L^{2}(\Omega)$.

Set $w=e^{-\psi} n, z=e^{\psi} p$ which is the so-called Boltzmann statistics. Then $\nabla n-$ $n \nabla \psi=e^{\psi} \nabla w, \nabla p+p \nabla \psi=e^{-\psi} \nabla z$. Multiplying (1.5)-(1.7) by a function $v \in V$, integrating over $\Omega$ and using Green's theorem and (1.11) we get

$$
\left.\begin{array}{l}
d(\psi, v)-\alpha(p-n+N, v)=0 \\
(\dot{n}, v)+\gamma_{n} \nu^{1}(\psi ; w, v)+(R(n, p), v)=0 \\
(\dot{p}, v)+\gamma_{p} \pi^{1}(\psi ; z, v)+(R(n, p), v)=0
\end{array}\right\} \quad \forall v \in V
$$

where

$$
\begin{gathered}
d(\psi, v)=\int_{\Omega} \nabla \psi \cdot \nabla v d x, \quad \nu^{1}(\psi ; w, v)=\int_{\Omega} \mu_{n}(x,\|\nabla \psi\|) e^{\psi} \nabla w \cdot \nabla v d x \\
\pi^{1}(\psi ; z, v)=\int_{\Omega} \mu_{p}(x,\|\nabla \psi\|) e^{-\psi} \nabla z \cdot \nabla v d x
\end{gathered}
$$

We are looking for $\psi, n, p$ such that

$$
\psi-\psi^{*}, n-n^{*}, p-p^{*} \in V, \quad n=e^{\psi} w, p=e^{-\psi} z, \psi\left(x^{0}, t\right)=0
$$


Of course, the conditions (1.12)-(1.14) remain, which means that $w(x, t)>0$, $z(x, t)>0$ on $\bar{Q}$. Without using Boltzmann statistics, the equations (1.16) and (1.17) have the form

$$
\begin{aligned}
& \left.\begin{array}{l}
(\dot{n}, v)+\gamma_{n} \nu^{2}(\psi ; n, v)+(R(n, p), v)=0 \\
(\dot{p}, v)+\gamma_{p} \pi^{2}(\psi ; p, v)+(R(n, p), v)=0
\end{array}\right\} \quad \forall v \in V, \\
& \nu^{2}(\psi ; n, v)=\int_{\Omega} \mu_{n}(x,\|\nabla \psi\|)[\nabla n-n \nabla \psi] \cdot \nabla v d x, \\
& \pi^{2}(\psi ; p, v)=\int_{\Omega} \mu_{p}(x,\|\nabla \psi\|)[\nabla p+p \nabla \psi] \cdot \nabla v d x .
\end{aligned}
$$

Remark 1.2. Let the data have the following properties:

(1) $\Gamma \in C^{2}, \operatorname{dist}\left(\Gamma^{1}, \Gamma^{2}\right)>0$;

(2) $N \in L^{q}(\Omega), q>m$;

(3) $d_{0}^{-1} \leqslant \mu_{s}(x, \xi) \leqslant d_{0}(=$ const $>0), s=n, p$;

(4) $n^{0}, p^{0} \in L^{2}(\Omega), n^{0}, p^{0}>0$ a.e. in $\Omega$;

(5) $\psi^{*} \in H^{2, q}(\Omega), q>m, n^{*}, p^{*} \in H^{1}(\Omega), n^{*}, p^{*}>0$ a.e. on $\Gamma^{1}$.

Then the problem (1.5)-(1.7), (1.10)-(1.14) can be formulated as follows: Find $\psi-\psi^{*} \in V \forall t \in(0, T), \psi\left(x^{0}, t\right)=0$ if $\Gamma^{1}=\varnothing, n-n^{*}, p-p^{*} \in L^{2}(0, T ; V) \cap$ $L^{\infty}\left(0, T ; L^{2}(\Omega)\right)$ satisfying $(1.12)-(1.14)$ and

$$
\begin{aligned}
& \forall t \in(0, T) \quad d(\psi, v)-\alpha(p-n+N, v)=0 \quad \forall v \in V, \\
& \left.\begin{array}{l}
\frac{d}{d t}(n, v)+\gamma_{n} \nu^{2}(\psi ; n, v)+(R(n, p), v)=0 \\
\frac{d}{d t}(p, v)+\gamma_{p} \pi^{2}(\psi ; p, v)+(R(n, p), v)=0
\end{array}\right\} \quad \text { in } \mathscr{D}^{\prime}((0, T)) \forall v \in V .
\end{aligned}
$$

We can easily prove that for any such $p$ and $n$,

$$
\frac{d n}{d t}=\frac{d\left(n-n^{*}\right)}{d t} \in L^{2}\left(0, T ; V^{\prime}\right) \quad \text { and } \quad \frac{d p}{d t}=\frac{d\left(p-p^{*}\right)}{d t} \in L^{2}\left(0, T ; V^{\prime}\right)
$$

( $V^{\prime}$ is the dual of $V$ ). Hence, as $n-n^{*}, p-p^{*} \in L^{2}(0, T ; V)$ we have (see, e.g., Girault-Raviart [3, p. 152]) $n-n^{*}, p-p^{*} \in C\left([0, T] ; L^{2}(\Omega)\right)$ and the initial conditions (1.12) make sense. Existence as well as unicity of the solution (not defined in this way) was proved by Mock [5] for the case that $\Gamma^{1}=\varnothing$ and $D_{s}, \mu_{s}$ are constant. Gajewski [2] proves existence and unicity for the case that the boundary conditions are of a general type and the diffusion coefficients and the mobilities are of the form

$$
D_{s}=U_{T} m_{s}, \quad m_{s}=\text { const }>0, \quad \mu_{s}(x,\|\nabla \psi\|)=m_{s}+M_{s}(x,\|\nabla \psi\|) .
$$

It is known that the discretization of the equations (1.5)-(1.7) by finite differences does not give good results if it is carried out in the standard way (see Scharfetter and Gummel [8, p. 73]). This is caused by extremely large values of $\|\nabla \psi\|$ close to junctions. Scharfetter and Gummel [8] proposed for the one-dimensional case to treat the equations (1.9) as differential equations in $n$ and $p$ with $\mathbf{J}_{n}, \mathbf{J}_{p}, \mu_{n}, \mu_{p}$ and $\nabla \psi$ assumed constant between mesh points. The scheme which they derived proved to be successful (see Mock [6] and references given there). We use the same idea: the quantities $\mathbf{J}_{n}, \mathbf{J}_{p}, \mu_{n}, \mu_{p}$ and $\|\nabla \psi\|$ are assumed constant on each element. Starting 
from this requirement we construct finite element approximations of the forms $\nu^{1}$, $\pi^{1}, \nu^{2}, \pi^{2}$ (see (1.22)). Then we introduce two fully discrete schemes for the solution of the problem (1.15)-(1.17). The first one is nonlinear, the other is partly linear and its motivation comes from results of a singular perturbation theory (see Section 4). We prove existence of positive solutions as well as uniqueness in the second case and show by means of Lemma 3.1 that the matrices appearing in these schemes have several important properties.

In the near future we want to investigate stability and convergence of both schemes. These are extremely difficult problems. The only results which were published, are, to our knowledge, results introduced in Mock's monograph [6]. Stability of a fully discrete scheme is there proved in one case only, namely for the scheme presented by Mock [5]. This scheme turns out to be the one-dimensional case of our nonlinear scheme (see Remark 3.1).

2. Discretization in Space. We cover $\bar{\Omega}$ by triangles or quadrilaterals if $\Omega \in R^{2}$ and by tetrahedrons or hexahedrons if $\Omega \subset R^{3}$, with nodes lying in $\bar{\Omega}$. We consider a family $\left\{\mathscr{T}_{h}\right\}$ of such partitions. Let $K$ (a closed set) denote an element of $\mathscr{T}_{h}$, $h_{K}=\operatorname{diam}(K), h=\max _{K \in \mathscr{T}_{h}} h_{K}$,

$$
\bar{\Omega}_{h}=\bigcup_{K \in \mathscr{T}_{h}} K \quad\left(\text { in general, } \Omega_{h} \neq \Omega\right),
$$

$\partial \Omega_{h}=\Gamma_{h}=\Gamma_{h}^{1} \cup \Gamma_{h}^{2} . \Gamma_{h}^{i}(i=1,2)$ are the parts of $\Gamma_{h}$ corresponding to $\Gamma^{i}$. We choose the partitions in such a way that all nodes of $\Gamma_{h}^{i}$ belong to $\Gamma^{i}(i=1,2)$ and that a boundary side and a boundary face, respectively, lie either in $\Gamma_{h}^{1}$ or in $\overline{\Gamma_{h}^{2}}$.

We consider linear polynomials on simplices and four-node and eight-node isoparametric elements on quadrilaterals and on hexahedrons, respectively. The geometry of the elements and the restrictions of the trial functions to $K$ can be expressed in this common way:

$$
\begin{gathered}
x=x(\xi) \equiv \sum_{j=1}^{d} x^{j} M_{j}(\xi), \quad \xi=\left(\xi_{1}, \ldots, \xi_{m}\right) \in \hat{K}, \\
\left.v(x)\right|_{K}=\sum_{j=1}^{d} v_{j} M_{j}(\xi), \quad \xi \in \hat{K} .
\end{gathered}
$$

Here $x^{j}=\left(x_{1}^{j}, \ldots, x_{m}^{j}\right)$ is a vertex of $K, d=3,4,8, v_{j}$ is the value $v\left(x^{j}\right)$ and $\hat{K}$ is the reference element. $\hat{K}$ is either the triangle $(0,0),(1,0),(0,1)$, or the tetrahedron $(0,0,0),(1,0,0),(0,1,0),(0,0,1)$ or the square $(-1,-1),(1,-1),(1,1),(-1,1)$ or the cube $(-1,-1,-1), \ldots,(-1,1,-1),(-1,-1,1), \ldots,(-1,1,1)$. Further, $M_{j}(\xi)$ (usually denoted by $\left.N_{j}(\xi)\right)$ are shape functions. We have for simplices

$$
M_{1}(\xi)=1-\xi_{1}-\cdots-\xi_{m}, \quad M_{2}(\xi)=\xi_{1}, \ldots, M_{m+1}(\xi)=\xi_{m}
$$

for quadrilaterals

$$
\begin{array}{ll}
M_{1}(\xi)=\frac{1}{4}\left(1-\xi_{1}\right)\left(1-\xi_{2}\right), & M_{2}(\xi)=\frac{1}{4}\left(1+\xi_{1}\right)\left(1-\xi_{2}\right), \\
M_{3}(\xi)=\frac{1}{4}\left(1+\xi_{1}\right)\left(1+\xi_{2}\right), & M_{4}(\xi)=\frac{1}{4}\left(1-\xi_{1}\right)\left(1+\xi_{2}\right)
\end{array}
$$

(concerning the remaining shape functions see, e.g., Zienkiewicz [9, p. 169]). 
To each partition from $\left\{\mathscr{T}_{h}\right\}$ we associate the finite-dimensional space

$$
V_{h}=\left\{v \in C^{0}\left(\bar{\Omega}_{h}\right) ;\left.v\right|_{K}=\sum_{j=1}^{d} v_{j} M_{j}(\xi),\left.v\right|_{\Gamma_{h}^{1}}=0\right\} .
$$

Also the space

$$
W_{h}=\left\{v \in C^{0}\left(\bar{\Omega}_{h}\right) ;\left.v\right|_{K}=\sum_{j=1}^{d} v_{j} M_{j}(\xi)\right\}
$$

will be needed.

As approximations of the functions $\psi, n, p, w, z$ we take functions $\Psi, N, P \in W_{h}$ and the functions $W, Z \in W_{h}$ defined by

$$
W_{j}=e^{-\Psi} N_{j}, \quad Z_{j}=e^{\Psi_{j}} P_{j} \quad \forall x^{j} \in \bar{\Omega}_{h} ;
$$

here the index $j$ denotes a value at the node $x^{j}$. (2.7) is in agreement with the Boltzmann statistics.

First we construct finite element analogues of the forms $\nu^{1}(\psi ; w, v)$ and $\pi^{1}(\psi ; z, v)$. We start from the requirement that the quantities $\mathbf{J}_{n}, \mathbf{J}_{p}, \mu_{n}, \mu_{p}$ and $\nabla \psi$ should be constant on each element. (1.9) is equivalent to

$$
\mathbf{J}_{n}^{*}=\mu_{n}(x,\|\nabla \psi\|) e^{\psi} \nabla w, \quad \mathbf{J}_{p}^{*}=-\mu_{p}(x,\|\nabla \psi\|) e^{-\psi} \nabla z .
$$

Let $\boldsymbol{\sigma}^{s}(s=n, p)$ be constant vectors which are the assumed approximations of $\mathbf{J}_{s}$ on $K$ and $\mu_{s}^{K}=\mu_{s}\left(x^{K},\left\|\nabla \psi\left(x^{K}\right)\right\|\right)$ where $x^{K}$ is the center of gravity of the element $K$. Then we require

$$
\boldsymbol{\sigma}^{n}=\mu_{n}^{K} e^{\psi} \nabla w, \quad \boldsymbol{\sigma}^{p}=-\mu_{p}^{K} e^{-\psi} \nabla z .
$$

Introducing the notation $\hat{\psi}(\xi)=\psi(x(\xi))$ where $x(\xi)$ is given by (2.1) and defining in the same way all the remaining functions, we derive

$$
\mu_{n}^{K} \nabla \hat{w}=e^{-\hat{\psi}} J^{T} \boldsymbol{\sigma}^{n}, \quad-\mu_{p}^{K} \nabla \hat{z}=e^{\hat{\psi}} J^{T} \boldsymbol{\sigma}^{p},
$$

$J$ being the Jacobian matrix of the mapping (2.1):

$$
J(\xi)=\frac{\partial\left(x_{1}, \ldots, x_{m}\right)}{\partial\left(\xi_{1}, \ldots, \xi_{m}\right)} .
$$

We consider first simplicial elements. Integrating the $j$ th components of (2.10) in the interval $[0,1]$ along the $\xi_{j}$ th axis and using a local notation $w_{1}, \ldots, w_{m+1}$ for values of $w$ at the vertices $x^{j}(j=1, \ldots, m+1)$ of $K$, we obtain

$$
\begin{aligned}
& \mu_{n}^{K}\left(\begin{array}{c}
w_{2}-w_{1} \\
\vdots \\
w_{m+1}-w_{1}
\end{array}\right) \\
& \quad=\operatorname{diag}\left(\int_{0}^{1} \exp \left[-\hat{\psi}\left(\xi_{1}, \ldots, 0\right)\right] d \xi_{1}, \ldots, \int_{0}^{1} \exp \left[-\hat{\psi}\left(0, \ldots, \xi_{m}\right)\right] d \xi_{m}\right) J^{T} \sigma^{n}, \\
& -\mu_{p}^{K}\left(\begin{array}{c}
z_{2}-z_{1} \\
\vdots \\
z_{m+1}-z_{1}
\end{array}\right) \\
& \quad=\operatorname{diag}\left(\int_{0}^{1} \exp \left[\hat{\psi}\left(\xi_{1}, \ldots, 0\right)\right] d \xi_{1}, \ldots, \int_{0}^{1} \exp \left[\hat{\psi}\left(0, \ldots, \xi_{m}\right)\right] d \xi_{m}\right) J^{T} \boldsymbol{\sigma}^{p} .
\end{aligned}
$$


In these equations we replace $w$ by $W, z$ by $Z$ and $\hat{\psi}$ by $\hat{\Psi}$, we compute the integrals and solve the resulting equations. We get

$$
\left\{\begin{array}{l}
\boldsymbol{\sigma}^{n}=\mu_{n}^{K} e^{\Psi_{1}}\left(J^{T}\right)^{-1} B^{K} \nabla \hat{W}=\mu_{n}^{K} e^{\Psi_{1}}\left(J^{T}\right)^{-1} B^{K} J^{T} \nabla W \\
\boldsymbol{\sigma}^{p}=-\mu_{p}^{K} e^{-\Psi_{1}}\left(J^{T}\right)^{-1} D^{K} \nabla \hat{Z}=-\mu_{p}^{K} e^{-\Psi_{1}}\left(J^{T}\right)^{-1} D^{K} J^{T} \nabla Z,
\end{array}\right.
$$

where

$$
\begin{cases}B^{K}=\operatorname{diag}\left(B\left(\Psi_{1}-\Psi_{2}\right), \ldots, B\left(\Psi_{1}-\Psi_{m+1}\right)\right), & B(\xi)=\xi\left(e^{\xi}-1\right)^{-1}, \\ D^{K}=\operatorname{diag}\left(D\left(\Psi_{1}-\Psi_{2}\right), \ldots, D\left(\Psi_{1}-\Psi_{m+1}\right)\right), & D(\xi)=e^{\xi} B(\xi)=B(-\xi) .\end{cases}
$$

Now, we define discrete analogues of the forms $\nu^{1}$ and $\pi^{1}$ for the case that $v=v^{k}$. Here $v^{k} \in V_{h}$ is the basis function associated with the node $x^{k}$ and defined by $v^{k}\left(x^{j}\right)=\delta_{j}^{k}, j, k=1, \ldots, q$ and $q$ is the number of all nodes not lying on $\Gamma_{h}^{1}$. The expressions (2.12) for $\sigma^{n}$ and $\sigma^{p}$ depend on the mapping (2.1). We choose this mapping in such a way that the node $x^{k}$ is mapped on the vertex $(0,0)$ and $(0,0,0)$, respectively, of the reference element $\hat{K}$. It is true that this way does not determine (2.1) uniquely; however, it suffices to a unique determination of the forms $\nu_{h}^{1}$ and $\pi_{h}^{1}$. Since we have (assuming $\Omega_{h}=\Omega$ )

$$
\nu^{1}\left(\psi ; w, v^{k}\right)=\sum_{K} \int_{K} \mathbf{J}_{n}^{*} \cdot \nabla v^{k} d x, \quad \pi^{1}\left(\psi ; z, v^{k}\right)=-\sum_{K} \int_{K} \mathbf{J}_{p}^{*} \cdot \nabla v^{k} d x,
$$

and since $v^{k}$ vanishes outside the set $S_{k}$, which is the union of all $K \in \mathscr{T}_{h}$ such that $x^{k}$ is a vertex of $K$, we define these discrete analogues in the following way:

$$
\left\{\begin{array}{l}
v_{h}^{1}\left(\Psi ; W, v^{k}\right)=\sum_{K \in S_{k}} \mu_{n}^{K} e^{\Psi_{k}} \int_{K}\left(J^{T}\right)^{-1} B^{K} J^{T} \nabla W \cdot \nabla v^{k} d x, \\
\pi_{h}^{1}\left(\Psi ; Z, v^{k}\right)=\sum_{K \in S_{k}} \mu_{p}^{K} e^{-\Psi_{k}} \int_{K}\left(J^{T}\right)^{-1} D^{K} J^{T} \nabla Z \cdot \nabla v^{k} d x .
\end{array}\right.
$$

For an arbitrary $v=\sum_{k=1}^{q} v_{k} v^{k}(x) \in V_{h}$ we define

$$
\nu_{h}^{1}(\Psi ; W, v)=\sum_{k=1}^{q} v_{k} \nu_{h}^{1}\left(\Psi ; W, v^{k}\right), \quad \pi_{h}^{1}(\Psi ; Z, v)=\sum_{k=1}^{q} v_{k} \pi_{h}^{1}\left(\Psi ; Z, v^{k}\right) .
$$

The forms $\nu_{h}^{1}$ and $\pi_{h}^{1}$ are symmetric with respect to the arguments $W, v$ and $Z, v$, respectively. We prove it only for $\nu_{h}^{1}$. We have to show that $\nu_{h}^{1}\left(\Psi ; v^{j}, v^{k}\right)=$ $\nu_{h}^{1}\left(\Psi ; v^{k}, v^{j}\right)$ for $j, k=1, \ldots, q$. Evidently, if $x^{j}$ and $x^{k}$ are not neighbors, then $\nu_{h}^{1}\left(\Psi ; v^{j}, v^{k}\right)=0=\nu_{k}^{1}\left(\Psi ; v^{k}, v^{j}\right)$. If $x^{j}$ and $x^{k}$ are neighbors, then consider all elements from $\mathscr{T}_{h}$ such that $x^{j}$ and $x^{k}$ are their vertices. Let $K$ be such an element. From the definition (2.14) we see that it suffices to prove

$$
\int_{K}\left(J_{k}^{T}\right)^{-1} B_{k} J_{k}^{T} \nabla v^{j} \cdot \nabla v^{k} d x=\int_{K}\left(J_{j}^{T}\right)^{-1} B_{j} J_{j}^{T} \nabla v^{k} \cdot \nabla v^{j} d x .
$$

Here $J_{s}, s=j, k$, are the Jacobian matrices of the mapping (2.1) which map $K$ on $\hat{K}$ in such a way that $x^{s}$ is mapped on the origin and $B_{s}=e^{\Psi_{s}} B^{K}$. The above equation is equivalent to

$$
\left(\nabla \hat{v}^{j}\right)^{T} B_{k} J_{k}^{-1}\left(J_{k}^{T}\right)^{-1} \nabla \hat{v}^{k}=\left(\nabla \hat{v}^{k}\right)^{T} B_{j} J_{j}^{-1}\left(J_{j}^{T}\right)^{-1} \nabla \hat{v}^{j},
$$

which can be verified in an elementary way. As the verification is lengthy, we omit it. 
Remark 2.1. It is evident that in the same way we can derive the discrete one-dimensional forms $\nu_{h}^{1}$ and $\pi_{h}^{1}$. These forms are equivalent to the finite-difference scheme proposed by Scharfetter and Gummel [8].

Remark 2.2. For $\Psi=0$ we have $B^{K}=D^{K}=I$. Hence, if $\mu_{n}=\mu_{p}=1$ the forms $\nu_{h}^{1}$ and $\pi_{h}^{1}$ are equal to $\int_{\Omega_{h}} \nabla W \cdot \nabla v d x$ and $\int_{\Omega_{h}} \nabla Z \cdot \nabla v d x$, respectively, which are the bilinear forms associated with the Laplace operator restricted to $V_{h}$.

Now, we introduce the forms $\nu_{h}^{1}$ and $\pi_{h}^{1}$ for the case of four- and eight-node isoparametric elements. We get them in the same way as above if we integrate (2.10) in the interval $[-1,1]$ along the parallels to the axes going through the points $(-1,-1)$ and $(-1,-1,-1)$, respectively. They become

$$
\begin{aligned}
& \left\{\begin{array}{l}
\nu_{h}^{1}\left(\Psi ; W, v^{k}\right)=\sum_{K \in S_{k}} \mu_{n}^{K} e^{\Psi_{k}}\left(J_{1}^{T}\right)^{-1} B^{K} \tilde{\nabla} W \cdot \int_{K} \nabla v^{k} d x, \\
\pi_{h}^{1}\left(\Psi ; Z, v^{k}\right)=\sum_{K \in S_{k}} \mu_{p}^{K} e^{-\Psi_{k}}\left(J_{1}^{T}\right)^{-1} D^{K} \tilde{\nabla} Z \cdot \int_{K} \nabla v^{k} d x,
\end{array}\right. \\
& \left\{\begin{array}{l}
\nu_{h}^{1}(\Psi ; W, v)=\sum_{k=1}^{q} v_{k} \nu_{h}^{1}\left(\Psi ; W, v^{k}\right) \\
\pi_{h}^{1}(\Psi ; Z, v)=\sum_{k=1}^{q} v_{k} \pi_{h}^{1}\left(\Psi ; Z, v^{k}\right)
\end{array}\right\} \forall v \in V_{h}
\end{aligned}
$$

Here,

$$
\begin{aligned}
& J_{1}=J(-1,-1), \quad \tilde{\nabla} W=\frac{1}{2}\left(\begin{array}{l}
W_{2}-W_{1} \\
W_{4}-W_{1}
\end{array}\right), \quad \tilde{\nabla} Z=\frac{1}{2}\left(\begin{array}{c}
Z_{2}-Z_{1} \\
Z_{4}-Z_{1}
\end{array}\right) \\
& B^{K}=\operatorname{diag}\left(B\left(\Psi_{1}-\Psi_{2}\right), B\left(\Psi_{1}-\Psi_{4}\right)\right) \\
& D^{K}=\operatorname{diag}\left(D\left(\Psi_{1}-\Psi_{2}\right), D\left(\Psi_{1}-\Psi_{4}\right)\right) \\
& \left.J_{1}=J(-1,-1,-1), \quad \tilde{\nabla} W=\frac{1}{2}\left(\begin{array}{l}
W_{2}-W_{1} \\
W_{4}-W_{1} \\
W_{5}-W_{1}
\end{array}\right), \quad \tilde{\nabla} Z=\frac{1}{2}\left(\begin{array}{c}
Z_{2}-Z_{1} \\
Z_{4}-Z_{1} \\
Z_{5}-Z_{1}
\end{array}\right)\right\} \text { if } m=3 . \\
& B^{K}=\operatorname{diag}\left(B\left(\Psi_{1}-\Psi_{2}\right), B\left(\Psi_{1}-\Psi_{4}\right), B\left(\Psi_{1}-\Psi_{5}\right)\right) \\
& D^{K}=\operatorname{diag}\left(D\left(\Psi_{1}-\Psi_{2}\right), D\left(\Psi_{1}-\Psi_{4}\right), D\left(\Psi_{1}-\Psi_{5}\right)\right)
\end{aligned}
$$

This time, the node $x^{k}$ is mapped by $(2.1)$ on the vertex $(-1,-1)$ and $(-1,-1,-1)$, respectively, of the reference element $\hat{K}$.

Finally, we derive $\nu_{h}^{2}(\Psi ; N, v)$ and $\pi_{h}^{2}(\Psi ; P, v)$ for the case of simplicial elements. The $j$ th component of the term $e^{\Psi_{1}} B^{k} \nabla \hat{W}$ in (2.12) is

hence

$$
\begin{aligned}
e^{\Psi_{1}} B\left(\Psi_{1}\right. & \left.-\Psi_{j+1}\right)\left(W_{j+1}-W_{1}\right) \\
& =e^{\Psi_{1}} B\left(\Psi_{1}-\Psi_{j+1}\right)\left[e^{-\Psi_{j+1}}\left(N_{j+1}-N_{1}\right)+\left(e^{-\Psi_{j+1}}-e^{-\Psi_{1}}\right) N_{1}\right] \\
& =D\left(\Psi_{1}-\Psi_{j+1}\right)\left(N_{j+1}-N_{1}\right)-\left(\Psi_{j+1}-\Psi_{1}\right) N_{1},
\end{aligned}
$$

$$
e^{\Psi_{1}} B^{K} \nabla \hat{W}=D^{K} \nabla \hat{N}-N_{1} \nabla \hat{\Psi}=D^{K} J^{T} \nabla N-N_{1} J^{T} \nabla \Psi
$$


and

$$
\left\{\begin{aligned}
\nu_{h}^{2}\left(\Psi ; N, v^{k}\right)= & \sum_{K \in S_{k}} \mu_{n}^{K} \int_{K}\left(J^{T}\right)^{-1} D^{K} J^{T} \nabla N \cdot \nabla v^{k} d x \\
& -\sum_{K \in S_{k}} \mu_{n}^{K} \int_{K} N_{k} \nabla \Psi \cdot \nabla v^{k} d x, \\
\nu_{h}^{2}(\Psi ; N, v)= & \sum_{k=1}^{q} v_{k} \nu_{h}^{2}\left(\Psi ; N, v^{k}\right) \quad \forall v \in V_{h} .
\end{aligned}\right.
$$

Similarly, we get

$$
\left\{\begin{aligned}
\pi_{h}^{2}\left(\Psi ; P, v^{k}\right)= & \sum_{K \in S_{k}} \mu_{p}^{K} \int_{K}\left(J^{T}\right)^{-1} B^{K} J^{T} \nabla P \cdot \nabla v^{k} d x \\
& +\sum_{K \in S_{k}} \mu_{p}^{K} \int_{K} P_{k} \nabla \Psi \cdot \nabla v^{k} d x, \\
\pi_{h}^{2}(\Psi ; P, v)= & \sum_{k=1}^{q} v_{k} \pi_{h}^{2}\left(\Psi ; P, v^{k}\right) \quad \forall v \in V_{h} .
\end{aligned}\right.
$$

3. A Nonlinear Scheme. In this section we restrict our attention to simplicial elements and to Neumann boundary conditions, i.e., we assume $\Gamma^{1}=\varnothing$. We will prove existence of the fully discrete approximate solution using, as in Mock [5], elementary degree theory. We need two things: 1) A priori estimates of the solution which we are able to get, as Mock [5], only for the case that $\Gamma^{1}=\varnothing$. 2) A maximum principle which we prove for simplicial elements under the condition, not too restrictive, that they are of acute type; for four- and eight-node isoparametric elements we are able to prove the maximum principle only for parallelograms and rectangular prisms, respectively.

Sufficient conditions for proving existence are as follows.

A: $N(x), n^{0}(x), p^{0}(x)$ are defined on $\bar{\Omega}, n^{0}(x)>0, p^{0}(x)>0$ on $\bar{\Omega}, \mu_{s}(x, \xi)$ $(s=n, p)$ are positive functions for $x \in \bar{\Omega}, \xi \in[0, \infty)$ which for each $x \in \bar{\Omega}$ are continuous for $\xi \in[0, \infty)$. Also, $\Omega_{h}$ must be such that all points $x^{K}$ lie in $\bar{\Omega}$.

The forms $d(\psi, v)$ and $(u, v)$ will be replaced by

$$
d_{h}(\Psi, v)=\int_{\Omega_{h}} \nabla \Psi \cdot \nabla v d x, \quad(u, v)_{h}=\sum_{K} I^{K}(u v) \quad \forall \Psi, u, v \in W_{h} .
$$

Here, $I^{K}$ is the quadrature formula

$$
I^{K}(F)=\frac{1}{m+1} \operatorname{meas}(K) \sum_{j=1}^{m+1} F_{j} .
$$

If $\mathbf{u}$ and $\mathbf{v}$ denote the vectors whose components are the values of $u$ and $v$ at the nodes, then

$$
(u, v)_{h}=\mathbf{u}^{T} M \mathbf{v}, \quad M=\left\{\left(v^{j}, v^{k}\right)_{h}\right\}_{j, k=1}^{q},
$$

where $v^{1}, v^{2}, \ldots, v^{q}$ are the basis functions: $v^{j} \in W_{h}, v^{j}\left(x^{k}\right)=\delta_{j}^{k}$. Evidently, the mass matrix $M$ is diagonal with positive diagonal elements (we denote them by $m_{j}$ ), hence $M$ is lumped by means of the quadrature formula $I^{K}$ (see, e.g., Zienkiewicz [ 9 , p. 537]). 
We have to replace the condition (1.13). We could restrict ourselves to the case $\Omega_{h}=\Omega$ and replace $N, n^{0}, p^{0}$ by $N_{h}, n_{h}^{0}, p_{h}^{0} \in W_{h}$ defined as follows: $\left(N_{h}, v\right)_{h}=$ $(N, v),\left(n_{h}^{0}, v\right)_{h}=\left(n^{0}, v\right),\left(p_{h}^{0}, v\right)_{h}=\left(p^{0}, v\right) \forall v \in W_{h}$ (then $\left(p_{h}^{0}-n_{h}^{0}+N_{h}, 1\right)_{h}=$ $\left.\int_{\Omega}\left[p^{0}-n^{0}+N\right] d x=0\right)$. Since computing exactly the $L^{2}$-products is impossible, we replace $N, n^{0}, p^{0}$ by their interpolates (i.e., functions from $W_{h}$ having the same values at all nodes as the original functions) and ask instead of (1.13)

$$
\left(p_{I}^{0}-n_{I}^{0}+N_{I}, 1\right)_{h}=0
$$

i.e.,

$$
\sum_{j=1}^{q} m_{j}\left[p_{j}^{0}-n_{j}^{0}+N_{j}\right]=0 .
$$

Let us remark that the choice of the discretization of the second term in (1.15) does not play any role in proving existence of the solution.

For simplicity, we consider an equally spaced partition of the interval $[0, T]$ : $t_{i}=i \Delta t, i=0,1, \ldots, r, r=T / \Delta t$. We denote by $\Psi^{i}, N^{i}, P^{i}, W^{i}, Z^{i} \in W_{h}\left(W^{i}\right.$ and $Z^{i}$ are determined by (2.7)) the approximations of $\psi^{i}, n^{i}, p^{i}, w^{i}, z^{i}$. In the sequel, the upper index $i$ will always mean a value at the time $t_{i}$. The forms $\nu^{1}$ and $\pi^{1}$ will be replaced by $\nu_{h}^{1}$ and $\pi_{h}^{1}$ and $\dot{n}\left(t_{i}\right), \dot{p}\left(t_{i}\right)$ by $\Delta N^{i} / \Delta t=\left(N^{i}-N^{i-1}\right) / \Delta t$ and by $\Delta P^{i} / \Delta t$, respectively.

Now, we are ready to introduce a fully discrete version of the problem (1.15)-(1.19), (1.12)-(1.14) when $\Gamma_{h}^{1}=\varnothing$.

Find $\Psi^{i}, N^{i}, P^{i} \in W_{h}$ satisfying

$$
\begin{aligned}
& \left.\begin{array}{l}
d_{h}\left(\Psi^{i}, v\right)-\alpha\left(P^{i}-N^{i}+N_{l}, v\right)_{h}=0, \quad \Psi_{j_{0}}^{i}=0 \\
\left(\Delta N^{i}, v\right)_{h}+\Delta t \gamma_{n} \nu_{h}^{1}\left(\Psi^{i} ; W^{i}, v\right)+\Delta t\left(R\left(N^{i}, P^{i}\right), v\right)_{h}=0 \\
\left(\Delta P^{i}, v\right)_{h}+\Delta t \gamma_{p} \pi_{h}^{1}\left(\Psi^{i} ; Z^{i}, v\right)+\Delta t\left(R\left(N^{i}, P^{i}\right), v\right)_{h}=0
\end{array}\right\} \begin{array}{l}
\forall v \in W_{h}, i= \\
1,2, \ldots, r,
\end{array} \\
& W_{j}^{i}=e^{-\Psi_{j}^{i}} N_{j}^{i}, \quad Z_{j}^{i}=e^{\Psi_{j}^{i}} P_{j}^{i}, \quad j=1,2, \ldots, \\
& N^{0}=n_{I}^{0}, \quad P^{0}=p_{I}^{0}, \\
& N^{i}, P^{i}>0 \text { on } \bar{\Omega}_{h} \text {. }
\end{aligned}
$$

Remark 3.1. If we discretize the one-dimensional problem in the above way and if we take $\gamma_{n} \mu_{n}=\gamma_{p} \mu_{p}=1$ we get a scheme identical with the difference scheme presented by Mock [5].

If $W \in W_{h}$ has a local extreme at a point from $\bar{\Omega}_{h}$ then evidently it attains it also at a node. $W$ attains a local maximum (minimum) at a node $x^{j}$ if and only if the values of $W$ at all neighboring nodes are not greater (smaller) than $W_{j}$. A simplicial partition is called of acute type if all angles of the triangles and all interior angles between faces of tetrahedrons, respectively, are not greater than $\frac{1}{2} \pi$.

The following lemma is a generalization of a lemma from Zlámal [10, p. 210]:

LEMMA 3.1. Let the given simplicial triangulation be of acute type. If $W \in W_{h}$ attains a local maximum (minimum) at a node $x^{j} \in \bar{\Omega}_{h}$ then

$$
\nu_{h}^{1}\left(\Psi ; W, v^{j}\right) \geqslant 0(\leqslant 0),
$$

where $v^{j}$ is the basis function associated with the node $x^{j}$. The same assertion is true for the form $\pi_{h}^{1}(\Psi ; Z, v)$. 
Proof. We consider first triangles and prove the lemma for the case of the maximum and of the form $\nu_{h}^{1}$. Since $\mu_{n}^{K} e^{\Psi_{j}}>0$, it is sufficient to prove that (see (2.14))

$$
\int_{K}\left(J^{T}\right)^{-1} B^{K} J^{T} \nabla W \cdot \nabla v^{j} d x \geqslant 0 \quad \forall K \in S_{j} .
$$

Displacement, rotation and reflection do not change the form of the above integrand. To see this, consider a rotation or reflection given by $\mathbf{x}=A \mathbf{y}, \mathbf{y}=\left(y_{1}, y_{2}\right)^{T}$, $A$ being the corresponding matrix. Then the mapping $\mathbf{y}(\xi)$ is equal to $A^{-1} \mathbf{x}(\xi)$ (see 2.11 ) and the new Jacobian matrix $J^{*}$ is equal to $A^{-1} J$. Hence, we get

$$
\begin{aligned}
\int_{K}\left(J^{T}\right)^{-1} B^{K} J^{T} \nabla_{x} W \cdot \nabla_{x} v^{j} d x \\
\quad=|\operatorname{det} A| \int_{K^{*}}\left(J^{T}\right)^{-1} B^{K} J^{T}\left(A^{T}\right)^{-1} \nabla_{y} W \cdot\left(A^{T}\right)^{-1} \nabla_{y} v^{j} d y \\
\quad=\int_{K^{*}}\left(J^{* T}\right)^{-1} B^{K} J^{* T} \nabla_{y} W \cdot \nabla_{y} v^{j} d y .
\end{aligned}
$$

We obtain the same result for a displacement $\mathbf{x}=\mathbf{y}+\mathbf{a}$.

We take such transformations that $(0,0)$ is the vertex of $K^{*}$ corresponding to $x^{j}$ and the remaining vertices are $\left(x_{2}, 0\right),\left(x_{3}, y_{3}\right), x_{2}>0, y_{3}>0$ (for the moment we denote the coordinates by $x, y$ and $x, y, z$, respectively). If we denote by $W_{1}, W_{2}$, $W_{3}$ the values of $W$ at these vertices then $W_{1} \geqslant W_{i}, i=2,3, v_{1}^{j}=1, v_{2}^{j}=v_{3}^{j}=0$ and we find that the integral in (3.10) is equal to

$$
\begin{aligned}
\frac{1}{2 x_{2} y_{3}}\left[\left(W_{1}-W_{2}\right) B(\right. & \left.\Psi_{1}-\Psi_{2}\right)\left(x_{3}^{2}+y_{3}^{2}-x_{2} x_{3}\right) \\
& \left.+\left(W_{1}-W_{3}\right) B\left(\Psi_{1}-\Psi_{3}\right) x_{2}\left(x_{2}-x_{3}\right)\right] .
\end{aligned}
$$

The function $B(\xi)$ is positive on the whole axis $-\infty<\xi<\infty$. As the triangulation is of acute type we have $x_{2} \geqslant x_{3} \geqslant 0$ and $x_{3}^{2}+y_{3}^{2}-x_{2} x_{3} \geqslant 0$ (easy to prove). Hence, (3.10) is true.

Now we consider tetrahedrons and prove that (3.10) is true. By displacement, rotation and reflection we achieve that the tetrahedron $K^{*}$ has vertices $A=(0,0,0)$, $B=\left(x_{2}, 0,0\right), C=\left(x_{3}, y_{3}, 0\right), D=\left(x_{4}, y_{4}, z_{4}\right), x_{2}>0, y_{3}>0, z_{4}>0$. We find that (3.10) is equal to

$$
\begin{aligned}
\frac{1}{b x_{2} y_{3} z_{4}}\left[\left(W_{1}-W_{2}\right) B\left(\Psi_{1}-\Psi_{2}\right) F_{1}+(\right. & \left.W_{1}-W_{3}\right) B\left(\Psi_{1}-\Psi_{3}\right) F_{2} \\
& \left.+\left(W_{1}-W_{4}\right) B\left(\Psi_{1}-\Psi_{4}\right) F_{3}\right],
\end{aligned}
$$

where

$$
\begin{aligned}
& F_{1}=\left(x_{3}^{2}+y_{3}^{2}-x_{2} x_{3}\right) z_{4}^{2}+\left(x_{4} y_{3}-x_{3} y_{4}\right)\left[y_{3} x_{4}+\left(x_{2}-x_{3}\right) y_{4}-x_{2} y_{3}\right], \\
& F_{2}=x_{2}\left(x_{2}-x_{3}\right) z_{4}^{2}+x_{2} y_{4}\left[y_{3} x_{4}+\left(x_{2}-x_{3}\right) y_{4}-x_{2} y_{3}\right], \\
& F_{3}=-x_{2} y_{3}\left[y_{3} x_{4}+\left(x_{2}-x_{3}\right) y_{4}-x_{2} y_{3}\right] .
\end{aligned}
$$

To finish the proof, it is sufficient to show that $F_{j} \geqslant 0, j=1,2,3$. We compute the cosine of the interior angle between the planes $B C D$ and $A C D$. We find that this cosine is equal to $F_{1} / D_{1}$, where $D_{1}>0$ and $\left|F_{1}\right| \leqslant D_{1}$. From acuteness there follows 
$F_{1} \geqslant 0$. We compute the cosine of the interior angle between the planes $B C D$ and $A B D$. We find that this cosine is equal to $F_{2} / D_{2}$, where $D_{2}>0$ and $\left|F_{2}\right| \leqslant D_{2}$. From acuteness there follows $F_{2} \geqslant 0$. From acuteness it follows that the point $\left(x_{4}, y_{4}\right)$ of the $x, y$-plane lies on the same side of the straight line going through $B C$ as the point $A$. Consequently $y_{3} x_{4}+\left(x_{2}-x_{3}\right) y_{4}-x_{2} y_{3} \leqslant 0$ and $F_{3} \geqslant 0$.

Remark 3.2. Lemma 3.1 is true for four-node isoparametric elements if these elements are parallelograms with diagonals making angles with sides which are not greater than $\frac{1}{2} \pi$. It is also true for eight-node isoparametric elements if these elements are rectangular prisms.

THEOREM 3.1. Let the conditions A and (3.4) be satisfied. If the triangulation is of acute type then the problem (3.5)-(3.8) has a solution.

Proof. Let $N^{i-1}, P^{i-1} \in W_{h}$ be positive functions on $\bar{\Omega}_{h}$, satisfying

$$
\left(P^{i-1}-N^{i-1}+N_{I}, 1\right)_{h}=0 .
$$

We consider the system

$$
\left\{\begin{array}{c}
d_{h}\left(\Psi^{\varepsilon}, v\right)-\alpha\left(P^{\varepsilon}-N^{\varepsilon}+N_{I}, v\right)_{h}=0, \quad \Psi_{j_{0}}^{\varepsilon}=0 \\
\left(N^{\varepsilon}, v\right)_{h}+\varepsilon \Delta t \gamma_{h} \nu_{h}^{1}\left(\Psi^{\varepsilon} ; W^{\varepsilon}, v\right)+\varepsilon \Delta t\left(R\left(N^{\varepsilon}, P^{\varepsilon}\right), v\right)_{h} \\
=\left(N^{i-1}, v\right)_{h} \\
\left(P^{\varepsilon}, v\right)_{h}+\varepsilon \Delta t \gamma_{p} \pi_{h}^{1}\left(\Psi^{\varepsilon} ; Z^{\varepsilon}, v\right)+\varepsilon \Delta t\left(R\left(N^{\varepsilon}, P^{\varepsilon}\right), v\right)_{h} \\
=\left(P^{i-1}, v\right)_{h}
\end{array}\right\} \quad \forall v \in W_{h}
$$

where

$$
W_{j}^{\varepsilon}=e^{-\Psi_{j}^{\epsilon}} N_{j}^{\varepsilon}, \quad Z_{j}^{\varepsilon}=e^{\Psi_{j}^{\varepsilon}} P_{j}^{\varepsilon}, \quad 0 \leqslant \varepsilon \leqslant 1 .
$$

Suppose, we prove that for $\varepsilon=1$ (3.12) has a solution $\Psi^{i}, N^{i}, P^{i}$ such that $N^{i}>0$, $P^{i}>0$ on $\bar{\Omega}_{h}$. This solution satisfies the condition $\left(P^{i}-N^{i}+N_{I}, 1\right)_{h}=0$ (put $v=1$ in the first equation in (3.12)). Taking successively $i=1,2, \ldots, r$, we prove the theorem.

First, we derive a priori estimates of $\Psi^{\varepsilon}, N^{\varepsilon}, P^{\varepsilon}$ supposing that the system (3.12) has a solution such that $N^{\varepsilon}>0, P^{\varepsilon}>0$ on $\bar{\Omega}_{h}$. We have

$$
0<\frac{\partial R(n, p)}{\partial n}<1, \quad 0<\frac{\partial R(n, p)}{\partial p}<1 \quad(n>0, p>0) .
$$

Therefore $-R(n, p)<-R(0,0)=\frac{1}{2}$. We denote by $\Psi^{\varepsilon}$ the vector $\left(\Psi_{1}^{\varepsilon}, \ldots, \Psi_{q}^{\varepsilon}\right)^{T}$ and use the same notation for the other functions. Since $\nu_{h}^{1}(\Psi ; W, 1)=\nu_{h}^{1}(\Psi ; 1, W)=0$ we get from the second of equations (3.12)

$$
\sum_{j=1}^{q} m_{j} N_{j}^{\varepsilon} \leqslant \sum_{j=1}^{q} m_{j} N_{j}^{i-1}+\frac{1}{2} \Delta t \sum_{j=1}^{q} m_{j}
$$

consequently $\left\|\mathbf{N}^{\varepsilon}\right\|_{\infty}<C_{1}\left[\left\|\mathbf{N}^{i-1}\right\|_{\infty}+1\right] \equiv C_{1}^{i-1}$, where $C_{1}^{i-1}$ is a constant not depending on $\varepsilon, \Psi^{\varepsilon}, \mathbf{N}^{\varepsilon}, \mathbf{P}^{\varepsilon}, 0 \leqslant \varepsilon \leqslant 1$ (it can and will depend on $h$ and $\Delta t$ ). The same argument proves $\left\|\mathbf{P}^{\varepsilon}\right\|_{\infty}<C_{2}^{i-1}$. The matrix form of the first equation in (3.12) is

$$
K \Psi^{\varepsilon}=\alpha M\left(\mathbf{P}^{\varepsilon}-\mathbf{N}^{\varepsilon}+\mathbf{N}_{I}\right),
$$


$K$ is symmetric, singular and $\lambda=0$ is a simple eigenvalue with $\mathbf{v}=(1, \ldots, 1)^{T}$ being the eigenvector. The necessary and sufficient condition $\left(P^{\varepsilon}-N^{\varepsilon}+N_{I}, 1\right)_{h}=0$ for solving (3.14) is satisfied, as we assume that a solution of (3.12) exists. We use the condition $\Psi_{j_{0}}^{\mathrm{F}}=0$, which means that we delete the $j_{0}$ th row and column in $K$, thereby obtaining a positive definite matrix $K_{0}$. Hence,

consequently,

$$
\left\|\Psi^{\varepsilon}\right\|_{\infty} \leqslant\left\|K_{0}^{-1}\right\|_{\infty}\left\|\alpha M\left(\mathbf{P}^{\varepsilon}-\mathbf{N}^{\varepsilon}+\mathbf{N}_{I}\right)\right\|_{\infty}
$$

$$
\left\|\Psi^{\varepsilon}\right\|_{\infty}<C_{3}\left[\left\|\mathbf{N}^{i-1}\right\|_{\infty}+\left\|\mathbf{P}^{i-1}\right\|_{\infty}+1\right]=C_{3}^{i-1} .
$$

Denoting $C_{0}^{i-1}=\max _{1 \leqslant j \leqslant 3} C_{j}^{i-1}$ we see that

$$
\left\|\mathbf{N}^{\varepsilon}\right\|_{\infty}<C_{0}^{i-1}, \quad\left\|\mathbf{P}^{\varepsilon}\right\|_{\infty}<C_{0}^{i-1}, \quad\left\|\Psi^{\varepsilon}\right\|_{\infty}<C_{0}^{i-1}, \quad 0 \leqslant \varepsilon \leqslant 1 .
$$

Let $\mathbf{X} \in R^{3 q}$ be the vector with components $\Psi_{1}^{\varepsilon}, \ldots, \Psi_{q}^{\varepsilon}, N_{1}^{\varepsilon}, \ldots, N_{q}^{\varepsilon}, P_{1}^{\varepsilon}, \ldots, P_{q}^{\varepsilon}$ and $Y$ the vector corresponding to the right-hand sides of the system (3.12). This is a nonlinear system, the left-hand side of which is a linear function of the parameter $\varepsilon$. Denote by $\mathbf{G}(\mathbf{X})$ and $\mathbf{F}(\mathbf{X})$ the vectors corresponding to the left-hand sides of (3.12) for $\varepsilon=0$ and $\varepsilon=1$, respectively. Then (3.12) is equivalent to

$$
(1-\varepsilon) \mathbf{G}(\mathbf{X})+\varepsilon \mathbf{F}(\mathbf{X})=\mathbf{Y} .
$$

Let us consider $\mathbf{G}(\mathbf{X})$ and $\mathbf{F}(\mathbf{X})$ as (continuous) mappings from $S \subset R^{3 q}$ into $R^{3 q}$, where $S$ is the domain

$$
\left\|\Psi^{\varepsilon}\right\|_{\infty}<C_{0}^{i-1}, \quad 0<N_{j}^{\varepsilon}<C_{0}^{i-1}, \quad 0<P_{j}^{\varepsilon}<C_{0}^{i-1}, \quad j=1, \ldots, q .
$$

For $\varepsilon=0$, (3.16) reduces to $\mathbf{G}(\mathbf{X})=\mathbf{Y}$. This system has just one solution in $S$. The second and third component of this solution are $\mathbf{N}^{i-1}, \mathbf{P}^{i-1}$ and the first is determined uniquely (owing to (3.11)) by (3.14) where we replace the index $\varepsilon$ by $i-1$. Therefore, if $\operatorname{deg}(\mathbf{G}, S, \mathbf{Y})$ means the degree of $\mathbf{G}$ with respect to $S$ and $\mathbf{Y}$ (see, e.g., Ortega, Rheinboldt [7, p. 154]) then $\operatorname{deg}(\mathbf{G}, S, \mathbf{Y})=1$. According to the Poincaré-Bohl theorem (see [7, p. 157]), $\operatorname{deg}(\mathbf{F}, S, \mathbf{Y})=\operatorname{deg}(\mathbf{G}, S, \mathbf{Y})$ if we prove that

$$
\mathbf{Y} \neq(1-\varepsilon) \mathbf{G}(\mathbf{X})+\varepsilon \mathbf{F}(\mathbf{X}) \quad \forall \mathbf{X} \in \partial S, 0 \leqslant \varepsilon \leqslant 1 .
$$

Then it follows from Kronecker's theorem (see [7, p. 161]) that the equation $\mathbf{F}(\mathbf{X})=\mathbf{Y}$ has a solution in $S$, i.e., (3.12) with $\varepsilon=1$ has a solution such that $N^{i}$, $P^{i}>0$ on $\bar{\Omega}_{h}$.

Suppose, $\mathbf{Y}=\left(1-\varepsilon_{0}\right) \mathbf{G}(\mathbf{X})+\varepsilon_{0} \mathbf{F}(\mathbf{X})$ for some $\mathbf{X} \in \partial S$ and some $\varepsilon_{0} \in[0,1]$. Since (3.15) holds as long as $N^{\varepsilon}>0, \quad P^{\varepsilon}>0$, necessarily at least one of the components of $\mathbf{N}^{\varepsilon_{0}}$ and $\mathbf{P}^{\varepsilon_{0}}$ is equal to zero. Let, say, $N_{j}^{\varepsilon_{0}}=0$. Because we have also $N_{k}^{\varepsilon_{0}} \geqslant 0, P_{k}^{\varepsilon_{0}} \geqslant 0, k=1, \ldots, q$, the function $N^{\varepsilon_{0}}$ attains a local minimum at $x^{j}$, consequently the same is true about $W^{\varepsilon_{0}}$. We set $v=v^{j}$ in the second equation in (3.12) and we get, on account of (3.9),

$$
m_{j} N_{j}^{i-1} \leqslant \varepsilon_{0} \Delta t m_{j} R\left(0, P_{j}^{\varepsilon_{0}}\right) \leqslant 0
$$

which is a contradiction.

Remark 3.3. Evidently, Theorem 3.1 remains true for isoparametric elements if we restrict oursleves to domains $\Omega$ which can be covered either by parallelograms having the property introduced in Remark 3.2 or by rectangular prisms. 
Remark 3.4. In practical computations the approximation $\left(N_{I}, v\right)_{h}$ of $(N, v)$ is not sufficient. On elements lying close to junctions we use a more accurate quadrature formula, or we cover such elements by smaller elements and use again the formula (3.2).

4. A Partly Linear Scheme. We consider again simplicial elements; however, the boundary conditions are of the general type (1.10) and (1.11) and $\Gamma^{1} \neq \varnothing . V_{h}$ is now the space

$$
V_{h}=\left\{v \in W_{h},\left.v\right|_{\Gamma_{h}^{1}}=0\right\}, \quad \Gamma_{h}^{1} \neq \varnothing,
$$

the Dirichlet condition reads

$$
\Psi_{j}^{i}=\psi^{*}\left(x^{j}\right), \quad N_{j}^{i}=n^{*}\left(x^{j}\right), \quad P_{j}^{i}=p^{*}\left(x^{j}\right) \quad \forall x^{j} \in \Gamma_{h}^{1}, i=1, \ldots, r .
$$

In addition, we have again (3.6), (3.7) and (3.8).

In the first equation of (3.5) we replace $N_{j}^{i}=e^{\Psi_{j}^{i}} W_{j}^{i}$ and $P_{j}^{i}=e^{-\Psi_{j}^{i}} Z_{j}^{i}$ by $e^{\Psi_{j}^{i}} W_{j}^{i-1}$ and $e^{-\Psi_{j}} Z_{j}^{i-1}$, respectively. This approximation is motivated by the fact that $w$ and $z$ are slow variables (see Markowich [4, Introduction]). After this modification the discrete Poisson equation has the form

$$
d_{h}\left(\Psi^{i}, v\right)+\alpha\left(e^{\Psi^{i}} W^{i-1}-e^{-\Psi^{i}} Z^{i-1}, v\right)_{h}=\alpha\left(N_{I}, v\right)_{h} \quad \forall v \in V_{h}, i=1, \ldots, r
$$

The remaining two equations differ from those in (3.5) in that the term $R\left(N^{i}, P^{i}\right)$ is replaced by $R\left(N^{i-1}, P^{i-1}\right)$. Hence,

$$
\left.\begin{array}{c}
\left(\Delta N^{i}, v\right)_{h}+\Delta t \gamma_{h} \nu_{h}^{1}\left(\Psi^{i} ; W^{i}, v\right) \\
=-\Delta t\left(R\left(N^{i-1}, P^{i-1}\right), v\right)_{h} \\
\left(\Delta P^{i}, v\right)_{h}+\Delta t \gamma_{p} \pi_{h}^{1}\left(\Psi^{i} ; Z^{i}, v\right) \\
=-\Delta t\left(R\left(N^{i-1}, P^{i-1}\right), v\right)_{h}
\end{array}\right\} \quad \forall v \in V_{h}, i=1, \ldots, r .
$$

We write the equations (4.2)-(4.4) in matrix form. We denote by $\Psi^{i}$ the vector $\left(\Psi_{1}^{i}, \ldots, \Psi_{q}^{i}\right)^{T}$ and use the same notation for the other functions. Then the equations are

$$
\begin{gathered}
K \Psi^{i}+\alpha M\left[E\left(\Psi^{i}\right) \mathbf{W}^{i-1}-E\left(\Psi^{i}\right)^{-1} \mathbf{Z}^{i-1}\right]=\alpha M \mathbf{N}_{I}+\mathbf{a} \\
A_{n} \mathbf{N}^{i}=M\left(\mathbf{N}^{i-1}-\Delta t \mathbf{R}^{i-1}\right)+\Delta t \mathbf{b}^{i-1}, \quad i=1, \ldots, r \\
A_{p} \mathbf{P}^{i}=M\left(\mathbf{P}^{i-1}-\Delta t \mathbf{R}^{i-1}\right)+\Delta t \mathbf{c}^{i-1}
\end{gathered}
$$

Here

$$
\begin{aligned}
& K=\left\{d_{h}\left(v^{j}, v^{m}\right)\right\}_{j, m=1}^{q}, \quad E\left(\Psi^{i}\right)=\operatorname{diag}\left(e^{\Psi_{1}^{i}}, \ldots, e^{\Psi_{q}^{i}}\right), \\
& A_{n}=B_{n} E\left(\Psi^{i}\right)^{-1}, \quad B_{n}=M E\left(\Psi^{i}\right)+\Delta t \gamma_{n} K_{n}\left(\Psi^{i}\right), \\
& K_{n}\left(\Psi^{i}\right)=\left\{\nu_{h}^{1}\left(\Psi^{i} ; v^{j}, v^{m}\right)\right\}_{j, m=1}^{q}, \\
& A_{p}=B_{p} E\left(\Psi^{i}\right), \quad B_{p}=M E\left(\Psi^{i}\right)^{-1}+\Delta t \gamma_{p} K_{p}\left(\Psi^{i}\right), \\
& K_{p}\left(\Psi^{i}\right)=\left\{\pi_{h}^{1}\left(\Psi^{i} ; v^{j}, v^{m}\right)\right\}_{j, m=1}^{q}, \\
& \mathbf{R}^{i-1}=\left(R\left(N_{1}^{i-1}, P_{1}^{i-1}\right)\right), \ldots,\left(R\left(N_{q}^{i-1}, P_{q}^{i-1}\right)\right)^{T},
\end{aligned}
$$

and the vectors $\mathbf{a}, \mathbf{b}^{i-1}, \mathbf{c}^{i-1}$ come from the Dirichlet conditions. 
Lemma 4.1. Let the triangulation be of acute type. Then the matrices $B_{s}(s=n, p)$ and $K$ are positive definite Stieltjes matrices and $A_{s}$ are $M$-matrices. In addition, $B_{s}$ are strictly diagonally dominant and $K$ is diagonally dominant (concerning the definition of $M$ - and Stieltjes matrices, see [7, p. 54]).

Proof. We restrict ourselves to the proof that $B_{n}$ is a positive definite strictly diagonally dominant Stieltjes matrix. First, we prove that the elements $b_{j m}$ of $B_{n}$ have the property $b_{j m} \leqslant 0$ if $j \neq m, b_{j j}>0$. We have $b_{j m}=\left(e^{\Psi^{i}} v^{j}, v^{m}\right)_{h}+$ $\Delta t \gamma_{n} \nu_{h}^{1}\left(\Psi^{i} ; v^{j}, v^{m}\right)$. The function $v^{j}$ has a local minimum at $x^{m}$ if $j \neq m$. Therefore, by Lemma 3.1, $b_{j m} \leqslant\left(e^{\Psi^{i}} v^{j}, v^{m}\right)_{h}=0$. Further, again by Lemma 3.1,

$$
b_{j j}=\left(e^{\Psi^{\prime}} v^{j}, v^{j}\right)_{h}+\Delta t \gamma_{n} \nu_{h}^{1}\left(\Psi^{i} ; v^{j}, v^{j}\right) \geqslant\left(e^{\Psi^{i}} v^{j}, v^{j}\right)_{h}>0 \text {. }
$$

Now, we prove that $K_{n}\left(\Psi^{i}\right)$ is diagonally dominant. Then $B_{n}$ is strictly diagonally dominant. To this end, let $x^{j}, j=q+1, \ldots, q^{*}$ be the remaining nodes, i.e., the nodes lying on $\Gamma_{h}^{1}$ and let us consider the matrix $K_{n}^{*}\left(\Psi^{i}\right)=\left\{v_{h}^{1}\left(\Psi^{i} ; v^{j}, v^{m}\right)\right\}_{j, m=1}^{q^{*}}$. Let $k_{j m}$ and $k_{j m}^{*}$ denote the elements of $K_{n}\left(\Psi^{i}\right)$ and $K_{n}^{*}\left(\Psi^{i}\right)$, respectively. Evidently, $k_{j m}=k_{j m}^{*}, j, m=1, \ldots, q$. As above, we prove by Lemma 3.1 that $k_{j j}^{*} \geqslant 0$, $k_{j m}^{*} \leqslant 0$ if $j \neq m$. Also, $k_{j m}^{*}=0$ if $x^{j}$ and $x^{m}$ are not neighbors. Further, we have $\nu_{h}^{1}\left(\Psi^{i} ; v^{j}, 1\right)=0$, consequently $\sum_{m=1}^{q^{*}} k_{j m}^{*}=0$. It follows that

$$
\begin{aligned}
\left|k_{j j}\right| & -\sum_{\substack{m=1 \\
m \neq j}}^{q}\left|k_{j m}\right| \\
& =\sum_{m=1}^{q} k_{j m}=\left\{\begin{array}{l}
\sum_{m=1}^{q^{*}} k_{j m}^{*}=0 \text { if } x^{j} \text { has no neighbor from } \Gamma_{h}^{1}, \\
\sum_{m=1}^{q^{*}} k_{j m}^{*}-\sum_{m_{j}} k_{j m_{j}}^{*} \geqslant 0 \text { if } x^{j} \text { has neighbors } x^{m_{j}} \text { from } \Gamma_{h}^{1} .
\end{array}\right.
\end{aligned}
$$

Since the form $\nu_{h}^{1}$ is symmetric, $B_{n}$ is symmetric. It has positive diagonal elements and is strictly diagonally dominant. It follows easily that all eigenvalues of $B_{n}$ are positive, hence $B_{n}$ is positive definite. It remains to prove that $B_{n}^{-1} \geqslant 0$. To this end, consider a vector $\mathbf{f} \geqslant 0$ and the vector $\mathbf{W}$ determined by $B_{n} \mathbf{W}=\mathbf{f}$, which is equivalent to

$$
\begin{gathered}
\left(e^{\Psi^{i}} W, v\right)_{h}+\Delta t \gamma_{n} \nu_{h}^{1}\left(\Psi^{i} ; W, v\right)=(g, v)_{h}, \quad W, g \in V_{h}, \\
W\left(x^{j}\right)=W_{j}, \quad g\left(x^{j}\right)=f_{j} m_{j}^{-1} .
\end{gathered}
$$

If $W_{j}=\min W$, then by Lemma 3.1, $\nu_{h}^{1}\left(\Psi^{i} ; W, v^{j}\right) \leqslant 0$, hence $e^{\Psi_{i}^{i}} W_{j} \geqslant g_{j} \geqslant 0$, consequently $\mathbf{W} \geqslant \mathbf{0}$, which means $B_{n}^{-1} \geqslant 0$.

Remark 4.1. For the two-dimensional case one can prove (assuming $\Gamma_{h}^{1} \neq \varnothing$ and the triangulation to be of acute type) that $K_{s}\left(\Psi^{i}\right)$ are positive definite diagonally dominant Stieltjes matrices.

THEOREM 4.1. Let the condition A be satisfied. If $\Delta t \leqslant 1$ and if the mesh is of acute type there is just one solution of (4.2)-(4.4) satisfying (4.1), (3.7) and (3.8).

Proof. Let us assume that $N^{i-1}$ and $P^{i-1}$ are positive. The components of the second term on the left-hand side of (4.5) are

$$
\alpha m_{j}\left[e^{\Psi_{i}^{i}} W_{j}^{i-1}-e^{-\Psi_{j}^{i} Z_{j}^{i-1}}\right]
$$


These are increasing functions of $\Psi_{j}^{i}$. As $K$ is a positive definite matrix, the mapping of $R^{q}$ into $R^{q}$ defined by the left-hand side of (4.5) is uniformly monotone. Therefore (see [7, p. 167]), (4.2) has just one solution.

From the regularity of $A_{s}, s=n, p$ ( $A_{s}$ are $M$-matrices by Lemma 4.1) there follows existence and uniqueness of $N^{i}$ and $P^{i}$. It remains to prove that $N^{i}$ and $P^{i}$ are positive. To this end, we consider the equations

$$
\begin{aligned}
& \left\{\begin{array}{l}
M \mathbf{N}^{\varepsilon}+\varepsilon \Delta t \gamma_{n} K_{n}\left(\Psi^{i}\right) \mathbf{W}^{\varepsilon}=M \mathbf{N}^{i-1}+\varepsilon \Delta t\left(-M \mathbf{R}^{i-1}+\mathbf{b}^{i-1}\right), \\
M \mathbf{P}^{\varepsilon}+\varepsilon \Delta t \gamma_{p} K_{p}\left(\Psi^{i}\right) \mathbf{Z}^{\varepsilon}=M \mathbf{P}^{i-1}+\varepsilon \Delta t\left(-M \mathbf{R}^{i-1}+\mathbf{c}^{i-1}\right),
\end{array}\right. \\
& \mathbf{N}^{\varepsilon}=E\left(\Psi^{i}\right) \mathbf{W}^{\varepsilon}, \quad \mathbf{P}^{\varepsilon}=E\left(\Psi^{i}\right)^{-1} \mathbf{Z}^{\varepsilon}, \quad \varepsilon \in[0,1] .
\end{aligned}
$$

The solutions exist and are unique (we apply the same argument as above). If we show that $N^{\varepsilon}$ and $P^{\varepsilon}$ are positive for $\varepsilon \in[0,1]$ then the proof is finished, since $N^{\varepsilon}=N^{i}, P^{\varepsilon}=P^{i}$ for $\varepsilon=1$. The variational form of (4.8) is

$$
\left\{\begin{array}{r}
\left(N^{\varepsilon}, v\right)_{h}+\varepsilon \Delta t \gamma_{n} \nu_{h}^{1}\left(\Psi^{i} ; W^{\varepsilon}, v\right)=\left(N^{i-1}, v\right)_{h}-\varepsilon \Delta t\left(R\left(N^{i-1}, P^{i-1}\right), v\right)_{h} \\
\left(P^{\varepsilon}, v\right)_{h}+\varepsilon \Delta t \gamma_{p} \pi_{h}^{1}\left(\Psi^{i} ; Z^{\varepsilon}, v\right)=\left(P^{i-1}, v\right)_{h}-\varepsilon \Delta t\left(R\left(N^{i-1}, P^{i-1}\right), v\right)_{h} \\
\forall v \in V_{h}
\end{array}\right.
$$

$N^{\varepsilon}$ and $P^{\varepsilon}$ depend continuously on $\varepsilon \in[0,1]$ and for $\varepsilon=0$ they are positive on $\bar{\Omega}_{h}$ (they are equal to $N^{i-1}$ and $P^{i-1}$, respectively). If they were not positive for $\varepsilon \in(0,1]$ there would exist $\varepsilon_{0}>0$ and $x^{j}$ such that either $N^{\varepsilon_{0}}\left(x^{j}\right)=0, N^{\varepsilon_{0}}(x) \geqslant 0$, $P^{\varepsilon_{0}}(x) \geqslant 0 \forall x \in \bar{\Omega}_{h}$ or vice versa. Consider the first case. Then $W^{\varepsilon_{0}}$ has a local minimum at $x^{j}$. Setting $v=v^{j}$ in (4.9) we get (by (3.13))

$$
\begin{aligned}
0 & \geqslant N_{j}^{i-1}-\varepsilon_{0} \Delta t R\left(N_{j}^{i-1}, P_{j}^{i-1}\right) \\
& =N_{j}^{i-1}-\varepsilon_{0} \Delta t\left[R\left(0, P_{j}^{i-1}\right)+\frac{\partial R\left(\vartheta N_{j}^{i-1}, P_{j}^{i-1}\right)}{\partial n} N_{j}^{i-1}\right]>\left(1-\varepsilon_{0} \Delta t\right) N_{j}^{i-1} \geqslant 0,
\end{aligned}
$$

which is not possible.

\section{Appendix}

In case of quadrilateral elements, Lemma 3.1 can be proved under the condition that the elements are convex and their diagonals make angles with the sides which are not greater than $\frac{1}{2} \pi$. However, in contradistinction to triangular elements, the forms $\nu_{h}^{1}$ and $\pi_{h}^{1}$ are not symmetric. The matrices $K_{s}(\Psi)$ must be defined as follows:

$$
K_{n}\left(\Psi^{i}\right)=\left\{\nu_{h}^{1}\left(\Psi^{i} ; v^{m}, v^{j}\right)\right\}_{j, m=1}^{q}, \quad K_{p}\left(\Psi^{i}\right)=\left\{\pi_{h}^{1}\left(\Psi^{i} ; v^{m}, v^{j}\right)\right\}_{j, m=1}^{q} .
$$

The matrices $B_{s}$ and $A_{s}(s=n, p)$ are defined as in the case of triangles. One can prove that $K_{s}\left(\Psi^{i}\right), B_{s}$ and $A_{s}$ are $M$-matrices with positive diagonal elements, and $K_{s}\left(\Psi^{i}\right)$ are diagonally and $B_{s}$ strictly diagonally dominant matrices.

Technical University

Obrancu miru 21

60200 Brno, Czechoslovakia

1. E. M. Buturla, P. E. Cottrell, B. M. Grossman \& K. A. Salsburg, "Finite-element analysis of semiconductor devices: The FIELDAY PROGRAM,” IBM J. Res. Develop., v. 25, 1981, pp. 218-231.

2. H. GAJEWSKI, "On existence, uniqueness and asymptotic behavior of solutions of the basic equations for carrier transport in semiconductors," Z. Angew. Math. Mech., v. 65, 1985, pp. 101-108.

3. V. Girault \& P. A. Raviart, Finite Element Approximation of the Navier-Stokes Equations, Springer-Verlag, Berlin and New York, 1979. 
4. P. A. Markowich, "A singular perturbation analysis of the fundamental semiconductor device equations,” SIAM J. Appl. Math., v. 44, 1984, pp. 896-928.

5. M. S. Mock, "An initial value problem from semiconductor device theory," SIAM J. Math. Anal., v. 5, 1974, pp. 597-612.

6. M. S. Mock, Analysis of Mathematical Models of Semiconductor Devices, Boole Press, Dublin, 1983.

7. J. M. Ortega \& W. C. Rheinboldt, Iterative Solution of Nonlinear Equations in Several Variables, Academic Press, New York, 1970.

8. D. L. Scharfetter \& H. K. Gummel, "Large signal analysis of a silicon Read diode oscillator," IEEE Trans. Electron. Devices, v. ED-16, 1969, pp. 64-77.

9. O. C. ZIENKIEWICZ, The Finite Element Method, McGraw-Hill, London, 1977.

10. M. Zlámal, "A finite element solution of the nonlinear heat equation," RAIRO Anal. Numér., v. 14, 1980, pp. 203-216. 